\title{
Necessidades humanas básicas alteradas em neonatos com cateter central de inserção periférica
}

\author{
Changed basic human needs in neonates with peripherally inserted central catheter \\ Necesidades humanas básicas alteradas en neonatos con catéter central insertado periféricamente
}

\author{
Nanete Caroline da Costa Prado'; Rebecca Stefany da Costa Santos"; Dhyanine Morais de Lima ${ }^{\prime \prime \prime}$; \\ Mariana Melo da Cruz Domingos Góis ${ }^{\prime V} ;$ Romanniny Hévillyn Silva Costavi Richardson Augusto Rosendo da Silva ${ }^{\text {VI }}$
}

\begin{abstract}
RESUMO
Objetivo: identificar as necessidades humanas básicas alteradas nos neonatos com cateter central de inserção periférica em Unidade de Terapia Intensiva Neonatal, à luz da Teoria das Necessidades Humanas Básicas. Método: estudo transversal de abordagem quantitativa, realizado com 108 recém-nascidos em uma maternidade do Nordeste brasileiro entre os meses de fevereiro e dezembro de 2017. O instrumento de coleta de dados utilizado foi um roteiro de anamnese e exame físico. Os resultados foram analisados por meio da Teoria de Wanda Horta. Resultados: foram identificadas dez necessidades alteradas, com destaque para as mais prevalentes: integridade cutâneo-mucosa, ambiente, nutrição, hidratação, oxigenação e atenção. Conclusão: a identificação das necessidades humanas básicas alteradas nos neonatos com cateter central de inserção periférica pode subsidiar a elaboração de um plano de cuidados baseado em evidências e direcionado para essa população.

Descritores: Terapia intensiva neonatal; cateterismo venoso central; teoria de enfermagem; processos de enfermagem.
\end{abstract}

\section{ABSTRACT}

Objective: to identify the changed basic human needs in neonates with peripherally inserted central catheter, in light of the Theory of Basic Human Needs. Method: cross-sectional study, conducted with 108 newborns in a maternity hospital in the Northeast of Brazil between February and December 2017. An anamnesis and physical examination script was used for data collection. The results were analyzed using the Wanda Horta Theory. Results: ten altered needs were identified, highlighting the most prevalent ones: mucosal skin integrity, environment, nutrition, hydration, oxygenation and attention. Conclusion: the identification of altered basic human needs in neonates with peripherally inserted central catheter may support the elaboration of a care plan based on scientific evidences and directed to this population..

Descriptors: Intensive care, neonatal; catheterization, central venous; nursing theory; nursing process.

\section{RESUMEN}

Objetivo: identificar las necesidades humanas básicas alteradas en recién nacidos con catéteres centrales insertados periféricamente en una Unidad de Cuidados Intensivos Neonatales, a la luz de la Teoría de las Necesidades Humanas Básicas. Método: estudio de enfoque cuantitativo, transversal, realizado con 108 recién nacidos en una maternidad en el nordeste de Brasil entre febrero y diciembre de 2017. El instrumento de recolección de datos utilizado fue un guión de historia y examen físico. Los resultados se analizaron utilizando la teoría de Wanda Horta. Resultados: se identificaron diez necesidades alteradas, destacando las más frecuentes: integridad de la piel, medio ambiente, nutrición, hidratación, oxigenación y atención. Conclusión: la identificación de necesidades humanas básicas alteradas en neonatos con catéter central insertado periféricamente puede apoyar la elaboración de un plan de atención basado en evidencias científicas y dirigido a esta población. Descriptores: Cuidado intensivo neonatal; cateterismo venoso central; teoría de enfermería; proceso de enfermería.

\section{INTRODUÇÃO}

O cateter central de inserção periférica (PICC), utilizado em larga escala nos serviços de pediatria e neonatologia, estabelece um acesso intravenoso seguro para a administração de medicamentos e nutrição que sustentam a vida. Este dispositivo pode ser inserido à beira leito e permanecer no sítio por várias semanas ${ }^{1}$. Além disso, apresenta baixo custo, contribui para a qualidade de vida e reduz a dor física e psicológica ${ }^{2}$. No Brasil, o primeiro relato de sua utilização se deu no ano de 1990 na área da neonatologia ${ }^{3}$.

'Enfermeira. Mestranda, Universidade Federal do Rio Grande do Norte. Residência Multiprofissional em Terapia Intensiva Neonatal, Maternidade Escola Januário Cicco. Natal, RN, Brasil. E-mail: caroline k16@hotmail.com

"Enfermeira. Especialista em Centro Cirúrgico e Central de Material Esterilizado, Faculdade Metropolitana de Ciências da Saúde. Natal, RN, Brasil. E-mail: rebecca.stefany@hotmail.com

I'Enfermeira. Mestranda, Universidade Federal do Rio Grande do Norte. Especialista em Unidade de Terapia Intensiva Neonatal e Pediátrica, Universidade Federal do Rio Grande do Norte. Natal, RN, Brasil. E-mail: dhyaninemorais@hotmail.com

IVEnfermeira. Especialista em Enfermagem do Trabalho e Unidade de Terapia Intensiva, Faculdade Metropolitana de Ciências e Tecnologia. Natal, RN, Brasil. E-mail: marimelogois@gmail.com

VEnfermeira. Mestre em Enfermagem. Doutoranda, Universidade Federal do Rio Grande do Norte. Enfermeira do Trabalho no Instituto Federal do Rio Grande do Norte e na Maternidade Escola Januário Cicco. Natal, RN, Brasil. E-mail: romanniny@yahoo.com.br

viProfessor Associado. Universidade Federal do Rio Grande do Norte, Departamento de Enfermagem. Natal, RN, Brasil. E-mail: rirosendo@hotmail.com 
A inserção do PICC consiste em sua introdução por meio de uma veia superficial ou profunda da extremidade, condução até o terço distal da veia cava superior ou proximal da veia cava inferior, e confirmação de sua localização por meio de radiografia. 0 procedimento é realizado, exclusivamente, por enfermeiro ou médico habilitados ${ }^{4}$.

O recém-nascido (RN) em uso do cateter em estudo demanda cuidados direcionados e sistematizados para que se alcance o sucesso na terapia endovenosa e sua consequente recuperação. Nesse sentido, para que a enfermagem atue eficientemente, necessita desenvolver sua metodologia de trabalho fundamentada em método científico. A esse método dá-se o nome de processo de enfermagem $(\mathrm{PE})^{4}$.

Historicamente, o PE favoreceu a emancipação da profissão. Sua utilização promove a qualificação profissional e valoriza a prática do cuidado, porém seu uso no Brasil ainda é incipiente. Desse modo, quando o PE é utilizado baseado em uma teoria, garante maior segurança na assistência de enfermagem, além de respaldo e caráter científico ${ }^{5}$.

É importante ressaltar que o RN com PICC apresenta várias necessidades, entre elas um acesso venoso seguro que garanta que a terapêutica medicamentosa seja realizada, o que irá contribuir no atendimento das necessidades nutricionais, imunológicas e de hidratação. Verifica-se, assim, a importância de se manter a integridade do cateter, a fim de evitar complicações hospitalares, tais como infecções da corrente sanguínea, lesões de pele e perdas acidentais.

Diante do cenário exposto e a fim de justificar o desenvolvimento do estudo foi realizada uma revisão de literatura com a seguinte questão norteadora: Quais as necessidades prioritárias de neonatos em uso do PICC?

Para tanto, realizou-se uma busca na literatura utilizando os descritores Teoria de enfermagem; Processos de enfermagem; Cuidados de enfermagem e Cateter venoso central, adotando como limite temporal os últimos cinco anos, nas bases de dados Literatura Internacional em Ciências da Saúde e Biomédica (Medline), Base de dados em Enfermagem (BDENF), Literatura Latino-Americana e do Caribe (Lilacs), Web of Science, SCOPUS e CINAHL.

Os resutados da revisão concentraram-se apenas na: técnica de iserção do cateter, cuidados com o seu manejo, educação permanente de Enfermeiros, avaliação da rede venosa de RNs, use de protocolos assistenciais, utilização do cateter como tecnologia de humanização da assistência e controle da dor ${ }^{6-15}$. No entanto, verificou-se escassez de pesquisas relacionadas as necessidades humanas básicas alteradas nos recém-nascidos que se submeteram à inserção do PICC, o que motivou a realização deste estudo.

Além disso, esta pesquisa é relevante pela contribuição que o enfermeiro pode fornecer na identificação das necessidades humanas básicas em desequilíbrio, como elemento para conduzir a elaboração de um plano de ações que direcione o atendimento das necessidades do neonato, visando qualificar a assistência de enfermagem, como também guiar o desenvolvimento de futuras investigações, contribuindo, dessa forma, para a construção do conhecimento e reconhecimento da enfermagem enquanto ciência.

Nesta conjuntura emergiu o seguinte questionamento: quais as necessidades humanas básicas alteradas nos recém-nascidos que se submeteram à inserção do cateter central de inserção periférica?

Desse modo, este estudo teve como objetivo identificar as necessidades humanas básicas alteradas nos neonatos com PICC, à luz da Teoria das Necessidades Humanas Básicas.

\section{REFERENCIAL TEÓRICO}

A enfermagem acumulou conhecimentos empíricos e dispõe de teorias que almejam dilucidar o seu universo. A teoria é importante como guia de ação, todavia ela não possui o objetivo de ditar como agir, mas sim o que acontecerá atuando-se de certa maneira. Na década de 1960, surgiram as primeiras teorias de enfermagem que buscaram estabelecer as bases de sua ciência ${ }^{4}$.

O PE ingressou no Brasil por Wanda de Aguiar Horta por meio de um modelo teórico intitulado Teoria das Necessidades Humanas Básicas. Seu modelo abrange leis que regem os fenômenos naturais: a lei do equilíbrio (homeostase), a lei da adaptação e a lei do holismo ${ }^{4}$.

A teoria das necessidades humanas básicas foi inspirada a partir da teoria da motivação humana, de Maslow. Nela, a enfermagem é a ciência e a arte de assistir o ser humano no atendimento de suas necessidades básicas. É fazer pelo ser humano aquilo que não está ao seu alcance; ajudar ou auxiliar quando parcialmente impossibilitado de se autocuidar; orientar ou ensinar, supervisionar e encaminhar a outros profissionais ${ }^{5}$. Horta criou uma nova classificação para as necessidades humanas, inspirada na denominação de João Mohana, nomeou-as em três níveis: psicobiológicas (relacionadas ao corpo físico, tais como oxigenação); psicossociais (referente à convivência com outros seres humanos) e psicoespirituais (derivadas dos valores e crenças individuais) ${ }^{16}$.

A teoria de Horta tem sido aplicada em diferentes contextos da assistência de enfermagem no Brasil. Um estudo que teve como objetivo descrever os problemas cotidianos vivenciados por pacientes com derivação urinária e relacioná-los com a Teoria das Necessidades Humanas Básicas de Wanda Horta, evidenciou que estes se sentiam 
afetados nas necessidades psicossociais e psicobiológicas, sendo a primeira mais manifesta. Além disso, destacou a importância da utilização desta teoria pela equipe de enfermagem na promoção de uma assistência integral, individualizada e sistematizada ao paciente com derivação urinária ${ }^{17}$.

Outro estudo recente cujo objetivo foi analisar o trabalho do enfermeiro no pré-natal de alto risco na atenção secundária, considerando os problemas de enfermagem e as necessidades humanas básicas das gestantes, identificou que as NHB psicobiológicas prevaleceram em relação às psicossociais. Além disso, a análise dos problemas de enfermagem e das necessidades alteradas dessa clientela permitiu identificar o trabalho do enfermeiro como parte constituinte de uma proposta assistencial para esta população ${ }^{18}$.

\section{MÉTOdo}

Trata-se de um estudo transversal, com abordagem quantitativa, realizada em uma maternidade escola situada no nordeste do Brasil. A escolha do local se deu por ser referência no atendimento materno-infantil de alto-risco na referida região.

A amostra foi composta de 108 neonatos em uso do PICC, utilizando-se a fórmula para populações finitas com nível de confiança de $95 \%$, erro amostral de $5 \%$ e população de 297 . A seleção dos pacientes foi obtida por meio da amostragem por conveniência do tipo consecutiva e as informações foram coletadas pela própria pesquisadora. A coleta de dados foi realizada entre os meses de fevereiro à dezembro de 2017.

Os critérios de inclusão para a participação no estudo foram: neonatos sem diagnóstico de coagulopatias e anomalias congênitas com perda de integridade da pele e que utilizaram PICC. Como critérios de exclusão: neonatos que tiveram o cateter inserido em instituição distinta à do estudo.

Respeitaram-se os aspectos éticos em pesquisa, como participação voluntária consentida pelos responsáveis pelos neonatos e assinatura prévia do Termo de Consentimento Livre e Esclarecido (TCLE). A pesquisa foi aprovada pelo Comitê de Ética, sob parecer número 1259309.

Utilizou-se um roteiro de anamnese e exame físico para coleta de dados, baseado na Teoria das Necessidades Humanas Básicas, o qual incluía dados socioeconômicos do neonato, avaliação neurológica, nutrição, eliminação, sono/repouso/atividade, relacionamentos, tolerância ao estresse, segurança/proteção e conforto, além de exame físico detalhado.

A avaliação do sistema nervoso do RN foi realizada através do exame físico geral, por meio da postura, movimentação espontânea, resposta ao manuseio e choro do RN, os quais são parâmetros importantes dessa avaliação. Os reflexos primitivos característicos do RN, mediados por mecanismos neuromusculares subcorticais, que se encontram desenvolvidos desde o período pré-natal, a exemplo da sucção e preensão palmar, também foram avaliados durante o exame físico ${ }^{19}$.

O instrumento foi submetido à validação do conteúdo e aparência por enfermeiros docentes, entre os meses de junho à dezembro de 2016. A amostra foi selecionada de forma intencional a partir da avaliação de seus currículos. Para tanto, realizou-se uma busca por meio da plataforma Lattes, do Conselho Nacional de Desenvolvimento Científico e Tecnológico (CNPq).

Os critérios de inclusão foram: ter pós graduação a nível de especialização ou residência em saúde da criança ou terapia intensiva neonatal com experiência na área assistencial de no mínimo dois anos, além de ter desenvolvido estudo publicado ou de conclusão de titulação (especialização, mestrado ou doutorado) relacionado à Sistematização da Assistência de Enfermagem, teorias de enfermagem e cateterismo venoso; já como critério de exclusão, informar no Currículo Lattes apenas o Trabalho de Conclusão do Curso de Graduação sobre a temática. 0 resultado foi de 14 especialistas.

Após a seleção dos especialistas, foi enviada uma carta convite via correio eletrônico, esclarecendo os objetivos da pesquisa e o TCLE. Assim, após a confirmação do aceite em participar da pesquisa e a assinatura no termo de consentimento livre e esclarecido, foi enviado o instrumento para análise por nove especialistas.

As sugestões propostas foram contempladas no instrumento, posteriormente aplicado, sob a forma de pré-teste, a dez pacientes submetidos ao cateter central de inserção periférica. Não houve necessidade de alterações, com os participantes do pré-teste sendo incluídos na amostra do estudo.

Ressalta-se que o processo de identificação das referidas necessidades alteradas e das histórias clínicas foi individualmente realizado por dois pesquisadores, a fim de possibilitar maior fidedignidade aos resultados obtidos. Os resultados que apresentaram concordância entre estes foram aceitos. Aqueles em que havia discordância entre os avaliadores, eram reavaliados em suas histórias clínicas até que se obtivesse um consenso. Além disso, os dados foram 
transferidos para o programa Microsoft Excel ${ }^{\circledR}$ para a realização da análise estatística descritiva e organização em tabelas com frequências absolutas e relativas.

\section{RESULTADOS E DISCUSSÃO}

Os neonatos eram, em sua maioria, nascidos de parto cesárea $(74,07 \%)$, do sexo masculino $(60,19 \%)$ e com baixo peso (30,56\%). Como principais causas de admissão na UTIN estiveram presentes: afecções respiratórias (79,62\%), incluindo desconforto respiratório precoce (DRP), síndrome do desconforto respiratório (SDR), asfixia perinatal, hipertensão pulmonar; prematuridade (78,70\%); cardiopatias (24,07\%); e sepse $(13,88 \%)$.

Observou-se que as principais indicações para a inserção foram: antibioticoterapia $(47,94 \%)$ e nutrição parenteral $(19,86 \%)$. A maioria dos cateteres $(59,25 \%)$ foi inserido logo nos primeiros dias de vida. Os enfermeiros insertores utilizaram, em sua maioria (58,33\%), até quatro tentativas para o sucesso na punção. Do total dos cateteres inseridos, $56,48 \%$ necessitaram de manobras de reposicionamento após a radiografia por terem suas pontas sido localizadas a nível intracardíaco. A inserção deu-se através do sistema introdutor Excalibur ${ }^{\circledR}$ destacável de calibre 1.9 Fr, comprimento de $50 \mathrm{~cm}$ e agulha introdutora.

Foram identificadas dez necessidades humanas básicas alteradas, sendo as mais prevalentes: integridade cutâneomucosa, ambiente, nutrição, hidratação, oxigenação e atenção. A Tabela 1 apresenta a distribuição das Necessidades Humanas Básicas alteradas em neonatos com cateter central de inserção periférica, divididas em necessidades psicobiológicas e psicossociais.

Tabela 1: Necessidades Humanas Básicas divididas em necessidades psicobiológicas e psicossociais em neonatos com cateter central de inserção periférica ( $n=108)$. Natal, 2017.

\begin{tabular}{lcc}
\hline Necessidades humanas básicas & $\mathbf{n}$ & $\mathbf{f ( \% )}$ \\
\hline Necessidades Psicobiológicas & & \\
Integridade cutâneo mucosa & 108 & 100,00 \\
Ambiente & 108 & 100,00 \\
Nutrição & 97 & 89,91 \\
Hidratação & 86 & 79,62 \\
Oxigenação & 86 & 79,62 \\
Cuidado corporal & 52 & 48,14 \\
Integridade física & 52 & 48,14 \\
Regulação & 51 & 34,25 \\
Sono e repouso & 37 & 34,25 \\
Necessidades Psicossociais & & \\
Atenção & 86 & 79,62 \\
\hline
\end{tabular}

Os principais diagnósticos dos neonatos estudados foram as afeç̧ões respiratórias, cardiopatias e infecções neonatais. Um estudo de revisão que objetivou identificar os distúrbios mais prevalentes responsáveis pela internação do RN em terapia intensiva observou que a prematuridade destacou-se como fator predominante, acompanhado do baixo peso ao nascer, das infecções e distúrbios respiratórios ${ }^{20}$.

A antecipação do parto pode acarretar em comprometimento ou intercorrências ao longo do crescimento do recém-nascido prematuro decorrente da imaturidade de órgãos ou sistema corporal. A Unidade de Terapia Intensiva Neonatal (UTIN) surge como alternativa de um ambiente com tecnologias avançadas e com intervenções cada vez mais modernas em prol de uma assistência de qualidade para a melhora do bebê prematuro ${ }^{21}$.

Entre as necessidades humanas básicas alteradas, a integridade cutâneo mucosa esteve entre a mais frequente, presente em todos os participantes do estudo, os quais apresentaram a quebra da barreira cutânea como sinal de desequilíbrio. A pele do neonato, caracterizada como fina e delicada, participa de reações fisiológicas e patológicas complexas, portanto, suas alterações são comuns nesse período. Estas características, associadas à imaturidade dos sistemas e à utilização de dispositivos médicos, imprescindíveis à sua sobrevivência, presdispõem ao risco de lesão da pele ${ }^{22}$. Nota-se que, apesar da redução considerável do número de punções venosas com o uso do PICC, o neonato teve sua integridade da pele rompida e, com isso, constitui-se uma porta de entrada para microorganismos. Logo, ao enfermeiro incumbe-se a responsabilidade de traçar metas e desenvolver protocolos a fim de evitar possíveis infecções, 
e para isso, é necessária a avaliação e a monitorização da inserção do dispositivo, juntamente com a observação contínua de qualquer eventualidade que possa interferir na saúde do bebê ${ }^{23}$.

Outra necessidade básica alterada identificada em toda a amostra foi o ambiente. O RN hospitalizado tem necessidade de manter-se em um meio ambiente livre de agentes agressores à vida com o objetivo de preservar a integridade psicobiológica. A ambientação extrauterina promove a intensificação de sua vulnerabilidade, o que não se limita ao aspecto físico. No ambiente de UTIN, muitos são os estímulos e procedimentos invasivos direcionados ao neonato, incluindo inserção de PICC, procedimento comumente realizado.

Considerando que a população de neonatos prematuros está sujeita a riscos, a atenção ao RN e sua avaliação clínica precisam ser estruturadas e sistemáticas. Sobremaneira, é fundamental o conhecimento das indicações e técnicas, além da escolha e utilização de material adequado para a realização dos procedimentos invasivos. A decisão da conduta exige cuidadosa avaliação dos riscos e benefícios dos procedimentos no cuidado neonatal ${ }^{24}$.

Nesse contexto, o PICC, que tem entre seus benefícios o de favorecer o bem-estar, por meio do manuseio mínimo e redução de estímulos dolorosos, está relacionado a resultados positivos em sua utilização, o que proporciona adequado gerenciamento dos riscos, maior conforto e segurança ${ }^{25}$.

No que concerne a venopunção periférica, esta é considerada um dos maiores avanços na área da saúde. Para tanto, é imprescindível a capacitação técnico-científica dos profissionais executantes, os quais devem respeitar as recomendações e evidências científicas construídas em vistas de um acesso seguro e eficaz, a fim de minimizar os riscos inerentes ao neonato ${ }^{26}$

A nutrição é uma condição que merece destaque, pois muitos neonatos apresentavam essa necessidade psicobiológica alterada devido ao baixo peso no nascimento decorrente da prematuridade. No ambiente extrauterino, o neonato encontra condições adversas daquelas fornecidas pela mãe e pela placenta; com destque para o consumo de energia que é maior no ambiente fora do útero materno. Então, para que o prematuro possa se aproximar das taxas de crescimento recomendadas é necessário um suporte nutricional adequado ${ }^{27}$.

Adequações energéticas e proteicas são alcançadas a partir do cálculo da razão entre as quilocalorias e proteínas obtidas e as prescritas, consideradas adequadas quando maiores ou iguais a $70 \%$ das necessidades calculadas. Ao longo das primeiras semanas de vida, foi reportado considerável déficit nutricional cumulativo e restrição do crescimento extrauterino de crianças prematuras. Uma das formas de recuperar o ganho de peso do RN prematuro é através da via parenteral por meio de um acesso venoso seguro, todavia, a deficiência na oferta energética por essa via está associada a um maior tempo para retabelecer o peso ao nascer e a falhas do crescimento pós-natal, o que pode, inclusive, explicar elevados percentuais de perda de peso ${ }^{28}$.

Em uma revisão da literatura foi observado que o início precoce da nutrição parenteral, imediatamente após o nascimento, associado à nutrição enteral trófica são fundamentais para que os RN de baixo peso possam apresentar uma curva de crescimento próxima a do período fetal ${ }^{29}$. Um estudo controlado randomizado recente mostrou melhor crescimento da cabeça em bebês muito prematuros durante o primeiro mês pós-natal com maior ingestão de proteínas e calorias ${ }^{30}$.

O início da nutrição tem associação com o crescimento dos prematuros. Além da restrição do crescimento, o início tardio da nutrição pode trazer outras consequências. Devido à imaturidade intestinal que dificulta a absorção de nutrientes, muitos prematuros dependem da nutrição parenteral por certo período ${ }^{27}$. Dessa forma, o PICC representa uma ferramenta primordial para a recuperação do peso por viabilizar a oferta da nutrição via parenteral.

A hidratação também foi uma necessidade psicobiológica com grandes alterações nos neonatos com PICC. Por meio do cateter em estudo, é possível ofertar aporte hídrico associado a eletrólitos e altas concentrações de glicose que garantem a sobrevida do RN. A necessidade hídrica infantil varia de acordo com a faixa etária e massa corpórea, a qual necessita ser ajustada para as suas condições clínicas, com observação constante do estado de hidratação e das perdas concomitantes ${ }^{31}$.

A avaliação diária do peso, débito urinário e balanço hídrico refletem uma estimativa do estado de hidratação do RN. O cálculo do balanço hídrico é a diferença entre a quantidade ingerida e administrada de líquidos e as perdas através da urina e das chamadas perdas insensíveis, que representam a eliminação de líquidos através da respiração e transpiração ${ }^{31}$. Nesse aspecto, o enfermeiro deve estabelecer como meta o equilíbrio hídrico e assim, realizar as intervenções necessárias como o controle hídrico, controle hidroeletrolítico, monitoração hídrica, avaliação da presença de edema e realização do balanço hídrico.

Outra necessidade psicobiológica contemplada no estudo foi a oxigenação. Como já mencionado anteriormente, o perfil de neonatos em UTIN é caracterizado, em sua maioria, pela imaturidade dos sistemas, principalmente o sistema respiratório. No presente estudo, grande parte dos RN que receberam o PICC apresentava algum grau de desconforto respiratório e faziam uso de terapia com oxigênio. 
Os cuidados neonatais têm se aperfeiçoado e melhorado progressivamente nos últimos anos, o que refletiu na sobrevida dos recém-nascidos, em particular dos prematuros. Nos nascimentos pré-termos, as doenças respiratórias surgem como o principal motivo de internação em unidade neonatal de cuidados intermediários e intensivos. Além disso, a terapêutica utilizada, a exemplo do suporte ventilatório, também tem o potencial de causar tais patologias durante o período de internação do $\mathrm{RN}^{32}$.

Destarte, a imaturidade pulmonar, associada à dificuldade de adaptação à vida extrauterina, resulta, geralmente, em terapia com oxigênio suplementar nos RN que apresentam dificuldade em realizar as trocas gasosas pulmonares. Como integrante da equipe multiprofissional, o enfermeiro que está em contato direto com o RN precisa avaliar constantemente o seu estado respiratório. É imprescindível que oriente e apoie a família e desenvolva um plano de cuidados baseado no processo de enfermagem, verificando e registrando os sinais vitais, assim como início e término da terapêutica ${ }^{33}$.

No que concerne ao aspecto psicossocial, a atenção foi uma necessidade identificada no presente estudo. Habituado à comunicação verbal, o ser humano é incentivado a ampliar sua consciência e sua visão para compreender a linguagem dos bebês, apoiada e traduzida em uma manifestação não verbal. Como forma de evidenciar a necessidade de atenção, o RN demonstra sinais como desconforto, irritabilidade, choro e alterações nos sinais vitais.

O recém-nascido é um ser competente, que dá sinais que guiam as atitudes dos pais e contribuem para estabelecer o vínculo afetivo. Além disso, o RN manifesta sintomas de estresse, tanto físico quanto comportamental, como resposta ao estímulo de enfrentamento do período de desenvolvimento extrauterino. Esse estímulo, por sua vez, tem relação direta com o meio ambiente ao qual o neonatal está inserido ${ }^{34}$.

Ao nascerem, os neonatos necessitam de condições favoráveis para um bom desenvolvimento psicomotor e mental. Tratando-se do RN prematuro, o qual apresenta ainda mais atrasos em relação à maturação neuropsicomotora quando comparado ao RN pré termo, a vivência de uma relação satisfatória e de amor é ainda mais necessária para um desenvolvimento mental saudável ${ }^{35,36}$.

Ao realizar o cuidado humanizado ao RN, é necessário analisar as necessidades apresentadas, com vistas também ao cuidado com a família. Revelar um ambiente de acolhimento para os mesmos, explicar a importância do envolvimento familiar e estabelecer o vínculo entre os pais e o bebê são ações que contribuem para a recuperação, acolhimento e conforto.

\section{CONCLUSÃO}

A pesquisa em questão proporcionoua identificação de dez necessidades humanasbásicas alteradas: integridade cutâneo mucosa, ambiente, nutrição, hidratação, oxigenação, cuidado corporal, integridade física, regulação, sono e repouso e atenção. Entretanto, cincoforam discutidas por serem predominantes naamostra estudada.

Comprovou-se que a utilização do modelo teórico de Horta pode subsidiar a sistematização da assistência de Enfermagem aos recém-nascidos com PICC, por contribuir com o enfermeiro para a elaboração de um plano de cuidados específico, direcionar as intervenções, aumentar a qualidade de vida desses neonatos e a visibilidade da enfermagem enquanto ciência. Além disso, a partir do conhecimento de tais respostas humanas, torna-se possível predizer e detectar as potenciais complicações.

Por fim, os limites do estudo estiveram relacionados ao perfil do local elegido, que pode não representar de forma fidedigna o perfil das demais instituições que prestam assistência ao RN com este tipo de cateter. Entretanto, esperase que os resultados do estudo contribuam para a padronização da linguagem específica de enfermagem e estimule outras pesquisas com o intuito de comparar os dados de distintas realidades.

\section{REFERÊNCIAS}

1. McCay AS, Elliott EC, Walden M. Videos in clinical medicine. PICC placement in the neonate. N Engl. j. med. [internet]. 2014 [cited 2019 Jul 20]; 370(11):e17.DOI: http://dx.doi.org/10.1056/NEJMvcm1101914

2. Gonçalves KG, Figueiredo JR, Oliveira SX, Davim RMB, Camboim JCA, Camboim FEF. Hospitalized child and the nursing team: opinion of caregivers. Rev enferm UFPE on line. [internet]. 2017 [cited 2019 Jul 20]; 11:2586-93. Available from: https://periodicos.ufpe.br/revistas/revistaenfermagem/article/download/23427/19115

3. Santo MKD, Takemoto D, Nascimento RG, Nascimento AM, Siqueira E, Duarte CT, et al. Cateteres venosos centrais de inserção periférica: alternativa ou primeira escolha em acesso vascular? J. Vasc. Bras. [internet]. 2017 [cited 2019 Jul 20]; 16(2):104-12. DOI: http://dx.doi.org/10.1590/1677-5449.011516

4. Horta WA. Processo de Enfermagem. Rio de Janeiro: Editora Guanabara Koogan LTDA; 2011.

5. Lins SMSB, Santo FHE, Fuly PSC, Garcia TR. Subset of ICNP ${ }^{\circledR}$ diagnostic concepts for patients with chronic kidney disease. Rev. Bras. enferm. [internet]. 2013 [cited 2019 Jul 20]; 66(2): 180-9. DOI: http://dx.doi.org/10.1590/s0034-71672013000200005 
6. Rangel RJM, Castro DS, Primo CC, Zandonade E, Christoffel MM, Amorim MHC. Cateter central de inserção periférica em neonato: revisão integrativa da literatura. J. res.: fundam. care. online [Internet]. 2016 [citado em 20 set 2019]; 8 (4): 5193 5202. DOI: http://dx.doi.org/10.9789/2175-5361.2016.v8i4.5193-5202

7. Bomfim JMS, Passos LS, Silva JC. Cateter central de inserção periférico: desafios e estratégias de enfermagem na manutenção do dispositivo. CuidArte Enferm. 2017 [cited 2019 Set 21] 11(1): 131-7. Available from:

http://www.webfipa.net/facfipa/ner/sumarios/cuidarte/2017v1/18\%20Artigo\%20Cateter_central\%20de\%20inser\%C3\%A7\%C3 \%A3०\%20perif\%C3\%A9rico\%20PICC.pdf

8. Borghesan NBA, Demitto MO, Fonseca LMM, Fernandes CAM,Castenaro RGS, Higarashi IH. Cateter venoso central de inserção periférica: práticas da equipe de enfermagem na atenção intensiva neonatal. Rev enferm UERJ. 2017 [cited 2019 Set 21] 25:e28143. DOI: http://dx.doi.org/10.12957/reuerj.2017.28143

9. Lui AML, Zily A, França AFO, Ferreira H, Toninato APC, Silva RMM. Cuidados e limitações no manejo do cateter central de inserção periférica em neonatalogia. Revista de Enfermagem do Centro-Oeste Mineiro. 2018 [cited 2019 Set 21] 8:e1918. DOI: http://dx.doi.org/10.19175/recom.v8i0.1918

10. Rangel RJM, Castro DS, Amorim MH, Zandonade E, Christoffel MM, Primo CC. Práticas de inserção, manutenção e remoção do Cateter Central de Inserção Periférica em neonatos. J. res.: fundam. care. online [Internet]. 2019 [cited 2019 Set 21] 11(2): 27884. DOI: http://dx.doi.org/10.9789/2175-5361.2019.v11i2.278-284

11. Jantsch LB, Neves ET, Arrué AM, Kegler JJ, Oliveira CR. Utilização do cateter central de inserção periférica em neonatologia. Revista Baiana de Enfermagem. 2014 [cited 2019 Set 21] 28 (3):244-51. Available from: https://portalseer.ufba.br/index.php/enfermagem/article/view/10109/

12. Silva MP, Bragato AG, Ferreira DO, Zago LB, Toffano SE, Nicolussi AC, et al. Bundle para manuseio do cateter central de inserção periférica em neonatos. Acta paul. enferm. [Internet]. 2019 [cited 2019 Set 21] 32( 3 ): 261-6. DOI : http://dx.doi.org/10.1590/1982-0194201900036

13. Oliveira CR, Neve ET, Rodrigues EC, Zamberlan KC, Silveira A. Peripherally inserted central catheter in pediatrics and neonatology: Possibilities of systematization in a teaching hospital. Esc. Anna Nery. 2014 [cited 2019 Set 21] 18(3):379-85. Available from: http://www.scielo.br/scielo.php?script=sci_arttext\&pid=S1414$81452014000300379 \&$ Ing=en\&nrm=iso\&tlng=en

14. Costa P, Kimura AF, Brandon DH, Paiva ED, Camargo PP. The development of a risk score for unplanned removal of peripherally inserted central catheter in newborns. Rev. Latino-Am. Enfermagem. 2015 [cited 2019 Set 21] 23(3):475-82. DOI: http://dx.doi.org/10.1590/0104-1169.0491.2578

15. Kegler JJ, Paula CC, Neves ET, Jantsch LB. Pain management in the use of the peripherally inserted central catheter in newborns. Esc Anna Nery. 2016 [cited 2019 Set 21] 20(4):e20160099. DOI: http://dx.doi.org10.5935/1414-8145.20160099

16. Freitas AAS, Coelho MJ. The Human Care Needs for Men Undergoing Oncological Surgical Treatment: Implications Towards the Nursing Profession. Rev Fundam Care Online. [internet]. 2019 [cited 2019 Jul 20]; 11 (n. esp): 481-7. DOI: http://dx.doi.org/10.9789/2175-5361.2019.v11i2.481-487

17. Ramos RCA, Costa CMA, Martins ERC, Clos AC, Francisco MTR, Spindola T. Patients with urinary derivations: an approach to basic human needs affected. Rev. enferm. UERJ [internet]. 2013 [cited 2019 Dec 4] 21(3):337-42. Available from: https://www.e-publicacoes.uerj.br/index.php/enfermagemuerj/article/view/7516/5439

18. Errico LSP, Bicalho PG, Oliveira TCFL, Martins EF. The work of nurses in high-risk prenatal care from the perspective of basic human needs. Rev Bras Enferm [Internet]. 2018 [cited 2019 Dec 4] 71(suppl 3):1335-43. DOI: http://dx.doi.org/10.1590/00347167-2017-0328

19. Ministério da Saúde (BR). Secretaria de Atenção à Saúde. Departamento de Ações Programáticas Estratégicas. Atenção à saúde do recém-nascido: guia para os profissionais de saúde. Brasília (DF): Ministério da Saúde; 2014.

20. Freitas MCN, Sousa AOB, Cabral SAAO, Alencar MCB, Guedes MSSE, Oliveira GF. Caracterização dos Recém-Nascidos Internados em Unidades de Terapia Intensiva. Id on Line Rev. Mult. Psic [internet]. 2018 [cited 2019 Jul 20]; 12(40). DOI: https://doi.org/10.14295/idonline.v12i40.1110

21. Almeida FA, Moraes MS, Cunha MLR. Taking care of the newborn dying and their families: Nurses' experiences of neonatal intensive care. Rev. Esc. Enferm. USP [internet]. 2016 [cited 2019 Jul 20]; 50(Spe):122-9. DOI: http://dx.doi.org/10.1590/S0080623420160000300018

22. Martins COA, Curado MAS. Escala de Observação do Risco de Lesão da Pele em Neonatos: validação estatística com recém-nascidos. Rev Enferm Referência. [internet]. 2017 [cited 2019 Jul 20]; 13(4):43-52. DOI: http://dx.doi.org/10.12707/RIV16082

23. Kegler JJ, Paula CC, Neves ET, Jantsch LB. Pain management in the use of the peripherally inserted central catheter in newborns. Esc. Anna Nery Rev. Enferm. [internet]. 2016 [cited 2019 Jul 20]; 20(4):e20160099. Available from: http://www.scielo.br/scielo.php?pid=S1414-81452016000400216\&script=sci_arttext\&tlng=en

24. Ministério da Saúde $(\mathrm{Br})$. Secretaria de Atenção à Saúde. Atenção humanizada ao recém-nascido: método canguru: manual técnico. Brasília (DF): Ministério da Saúde ; 2017.

25. Macedo TR, Guimarães GP. Percepções das enfermeiras neonatologistas sobre as causas da retirada do cateter central de inserção periférica. [internet]. 2015 [cited 2019 Jul 20]; 6(2):1408-417. Available from: https://periodicos.unb.br/index.php/rgs/article/view/2956

26. Melo EM, Aragão AL, Pessoa CMP, Lima FET, Barbosa IV, Studart RMB, et al. Care provided by nursing staff during the peripheral venipuncture procedure. Rev. enferm. UFPE on line [internet]. 2015 [cited 2019 Jul 20]; 9(3):1022-30. Available from: http://www.repositorio.ufc.br/bitstream/riufc/10892/1/2015_art_ivbarbosa.pdf 
27. Damasceno JR, Silva RCC, Ximenes Neto FRG, Ferreira AGN, Silva ASR, Machado MMT. Nutrition in premature and low birth weight newborns: an integrative review. Rev. Soc. Bras. Enferm. Ped [internet]. 2014 [cited 2019 Jul 20]; 14(1):40-6. Available from: https://sobep.org.br/revista/images/stories/pdf-revista/vol14-n1/v14_n1_artigo_revisao_2.pdf

28. lacobelli S, Viaud M, Lapillonne A, Robillard P, Gouyon J, Bonsante F. Nutrition practice, compliance to guidelines and postnatal growth in moderately premature babies: the NUTRIQUAL French survey. BMC. pediatr [internet]. 2015 [cited 2019 Jul 20]; 15(1). DOI: https://doi.org/10.1186/s12887-015-0426-4

29. Maas C, Poets CF, Franz AR. Avoiding postnatal undernutrition of VLBW infants during neonatal intensive care: evidence and personal view in the absence of evidence. Arch. dis. child. fetal neonatal ed [internet]. 2015 [cited 2019 Jul 20]; 100:F76-F81. DOI: https://doi.org/10.1136/archdischild-2014-306195

30. Morgan C, McGowan P, Herwitker S, et al. Postnatal head growth in preterm infants: a randomized controlled parenteral nutrition study. Pediatrics [internet]. 2014 [cited 2019 Jul 20]; 133:e120-8. DOI: https://doi.org/10.1542/peds.2013-2207

31. ChoongK, Bohn D. Maintenence parenteral fluids in the critically ill child. J Pediatr [internet]. 2007 [cited 2019 Jul 20]; 83(2):S310. DOI: https://doi.org/10.2223/JPED.1614

32. Sousa NFC, Bonfim SFSF, Vasconcelos MGL, Bezerra JLO, Silva DVC, Leal LP. Prevalence of nasal septum injury in premature infants using nasal prongs. Rev. Esc. Enferm. USP [internet]. 2013 [cited 2019 Jul 20]; 47(6):1285-90. DOI: http://dx.doi.org/10.1590/S0080-623420130000600005.

33. Borges JPA. Monitorização da oximetria de pulso em recém-nascidos: atuação do enfermeiro nas unidades neonatais. Rev. enferm. atenção saúde [internet]. 2013 [cited 2019 Jul 20];2(3):106-14. Available from: http://seer.uftm.edu.br/revistaeletronica/index.php/enfer/article/view/595

34. Habersaat S, Borghini A, Faure N, Nessi J, Forcada-Guex M, Pierrehumbert B, et al. Emotional and neuroendocrine regulation in very preterm and full-term infants at six months of age. Eur J Dev Psychol [internet]. 2013. [cited 2019 Dec 4] 10:691-706. DOI: https://doi.org/10.1080/17405629.2013.787924

35. Silva CMS, Dantas JC, Souza FML, Silva RAR, Lopes TRG, Carvalho JBL. Feelings experienced by postpartum women in achieving the first bath of the newborn in rooming. O Mundo da Saúde [internet]. 2015 [cited 2019 Dec 5] 39(3):279-86. DOI: http://dx.doi.org/10.15343/0104-7809.20153903279286

36. Romeo DM, Brogna C, Sini F, Romeo MG, Cota F, Ricci D. Early psychomotor development of low-risk preterm infants: Influence of gestational age and gender. Eur J Paediatr Neurol [internet]. 2016 [cited 2019 Dec 4] 20(4):518-23. DOI: http://dx.doi.org/10.1016/j.ejpn.2016.04.011 